\title{
Food patterns associated with blood lipids are predictive of coronary heart disease: the Whitehall II study
}

\author{
Sarah A. McNaughton ${ }^{1 *}$, Gita D. Mishra ${ }^{2}$ and Eric J. Brunner ${ }^{2}$ \\ ${ }^{1}$ School of Exercise and Nutrition Sciences, Deakin University, 221 Burwood Highway, Melbourne 3125, Australia \\ ${ }^{2}$ Department of Epidemiology and Public Health, University College London, 1-19 Torrington Place, London WC1E 6BT, UK
}

(Received 1 August 2008 - Revised 12 December 2008 - Accepted 23 December 2008 - First published online 30 March 2009)

Analysis of the epidemiological effects of overall dietary patterns offers an alternative approach to the investigation of the role of diet in CHD. We analysed the role of blood lipid-related dietary patterns using a two-step method to confirm the prospective association of dietary pattern with incident CHD. Analysis is based on 7314 participants of the Whitehall II study. Dietary intake was measured using a 127-item FFQ. Reduced rank regression (RRR) was used to derive dietary pattern scores using baseline serum total and HDL-cholesterol, and TAG levels as dependent variables. Cox proportional hazard regression was used to confirm the association between dietary patterns and incident CHD ( $n$ 243) over 15 years of follow-up. Increased CHD risk (hazard ratio (HR) for top quartile: 2.01 (95\% CI 1.41, 2.85) adjusted for age, sex, ethnicity and energy misreporting) was observed with a diet characterised by high consumption of white bread, fried potatoes, sugar in tea and coffee, burgers and sausages, soft drinks, and low consumption of French dressing and vegetables. The diet-CHD relationship was attenuated after adjustment for employment grade and health behaviours (HR for top quartile: 1.81; $95 \%$ CI 1.26, 2.62), and further adjustment for blood pressure and BMI (HR for top quartile: $1.57 ; 95 \%$ CI 1.08, 2.27). Dietary patterns are associated with serum lipids and predict CHD risk after adjustment for confounders. RRR identifies dietary patterns using prior knowledge and focuses on the pathways through which diet may influence disease. The present study adds to the evidence that diet is an important risk factor for CHD.

Dietary patterns: Lipids: Coronary heart disease: Prospective cohort studies: Whitehall II study

Investigation of the role of diet in CHD may focus on individual nutrients, food groups or overall dietary patterns. Disentangling the effects of correlated nutrient intakes such as MUFA and PUFA is problematic, likewise, those of food groups such as wholegrain cereals, fruit and vegetables ${ }^{(1)}$. Analysis of the epidemiological effects of overall dietary patterns offers an alternative approach ${ }^{(2)}$. Several a posteriori statistical methods have been used to determine dietary patterns $^{(2,3)}$. Reduced rank regression (RRR) is particularly useful as a hypothesis-driven method for confirming pathways through which diet may act to alter chronic disease risk ${ }^{(4)}$. RRR combines multivariate dietary pattern analysis with existing knowledge of diet-disease relationships ${ }^{(5)}$. In the first step, factor loadings for food groups are derived in an RRR model with intermediate disease markers, such as biomarkers of disease process, as one or more dependent variables. In the second step, the relationship between dietary pattern scores and disease outcome is tested. This methodology has been used to study dietary influences on obesity ${ }^{(6)}$, CVD $^{(7-10)}$, diabetes ${ }^{(11,12)}$ and all-cause mortality ${ }^{(13)}$.

Studies of diet and CHD using RRR methods have focused on pathways based on the fat, carbohydrate and fibre density of the diet ${ }^{(10)}$, plasma homocysteine, folate and vitamin $\mathrm{B}_{12}^{(8)}$ and HDL-cholesterol, LDL-cholesterol, lipoprotein(a), C-peptide and C-reactive protein ${ }^{(7)}$. While Hoffmann et al. ${ }^{(7)}$ utilised HDL- and LDL-cholesterol as dependent variables, their study used a case-control design with potential measurement bias relating to the recall of dietary intake and the timing of the biomedical risk factor measurement. In addition, they incorporated markers of other risk-factor pathways and did not focus on specific lipid-related dietary patterns. Research exploring the relationship between dietary patterns and lipids using RRR methods focuses on the pathways through which diet may influence CHD.

The aim of the present study was to explore the link between dietary patterns associated specifically with blood lipid levels - serum total and HDL-cholesterol and TAG and risk of verified incident coronary events over 15 years of follow-up in a large prospective cohort of men and women.

\section{Materials and methods}

\section{Study population}

Men and women aged 35-55 years were recruited to the Whitehall II study in 1985-8 (phase 1) from twenty Civil Service departments in London with a $73 \%$ response rate ( $n$ 10308). Full details of the study are reported elsewhere $^{(14,15)}$. Medical examinations and self-report questionnaires were repeated at 5-yearly intervals and the cohort

Abbreviations: EE, energy expenditure; EI, energy intake; RRR, reduced rank regression.

* Corresponding author: Dr Sarah A. McNaughton, fax +61 39244 6017, email sarah.mcnaughton@deakin.edu.au 
completed postal questionnaires between each clinic visit. At phase 3 (1991-3), 8104 participants attended the screening clinic. Ethical approval was obtained from the University College London Medical School Committee on the Ethics of Human Research. Informed consent was obtained at phase 1 and renewed at each contact. This analysis is based on data from 7314 participants that completed an FFQ at phase 3, who were fasted (duration of fasting $>5 \mathrm{~h}$ ) at the time of blood sample collection, had no history of CHD at the phase 3 screening and had complete data for all covariates.

\section{Outcome ascertainment}

Participants were flagged by the National Health Service Central Registry, which notified us of the date and cause of all deaths up to 31 July 2006. Deaths were classified as coronary if International Classification of Diseases (ICD)-9 codes 410-414 or ICD10 codes I20-I25 were present on the death certificate. Cases of non-fatal myocardial infarction were identified from twelve-lead electrocardiograms obtained at study phases 3,5 and 7 and questionnaire items on chest pain and doctor's diagnosis. Details of physician investigations, diagnoses and interventions were sought from medical records for all potential cases for final ascertainment. Classification, following WHO Monitoring of Trends and Determinants in Cardiovascular Disease (MONICA) methods, was carried out independently by two trained coders, with adjudication in the event of disagreement. Self-report in the absence of verification was not classified as myocardial infarction. Prevalent cases of myocardial infarction were excluded from the analysis. Participants' date of censoring depended on the last attended phase: 30 September 2004 for those who attended phase 7 .

\section{Dietary intake}

Dietary intake was assessed using a 127-item FFQ. Participants were asked to report the frequency of consumption of each food during the previous year. Standard portion sizes were specified for each food with response options ranging from 'never or less than once per month' to 'six or more times per day'. Responses were converted to food intake in $\mathrm{g} / \mathrm{d}$ for analysis. The questionnaire was previously validated in this cohort among 457 males and 403 females aged 39-61 years using $7 \mathrm{~d}$ food diaries ${ }^{(16)}$. Spearman correlation coefficients for energy-adjusted nutrient intakes ranged from 0.35 ( $\beta$-carotene) to 0.78 (alcohol) for men and 0.33 (vitamin E) and 0.83 (alcohol) for women.

Total energy intake (EI) was evaluated by examining the ratio EI:estimated energy expenditure (EE), reflecting energy misreporting. The ratio EI:EE will be 1 if there is no energy misreporting and less than 1 if there is underreporting ${ }^{(17)}$. EE was based on estimated $\mathrm{BMR}^{(18)}$ and a physical activity level of 1.55 (sedentary) was assumed for this cohort of office workers. The energy cost of reported leisure time physical activity was added using metabolic equivalent (MET) values for $\mathrm{EE}$ per $\mathrm{kg}$ body weight ( $3 \mathrm{MET} / \mathrm{h}$ moderate activity, 5 $\mathrm{MET} / \mathrm{h}$ vigorous activity) ${ }^{(19)}$. Fifty-five participants $(0.75 \%)$ with a $\log \mathrm{EI}: \mathrm{EE}$ value outside $3 \mathrm{SD}$ of the $\log$ mean were excluded from the analysis.

The food and beverage items on the FFQ were aggregated into seventy-one food groups on the basis of nutrient content, cooking and preparation methods and consistency with groupings used in other studies of dietary patterns and $\operatorname{CVD}^{(2,4,20,21)}$ and the UK National Diet and Nutrition Survey ${ }^{(22)}$. In addition, single food items that were hypothesised to represent specific eating behaviours were retained ${ }^{(23-25)}$. As alcohol intake has a distinctive U-shaped relationship with CVD and all-cause mortality $^{(26)}$, we excluded alcoholic beverages from the dietary pattern analysis and treated them as a separate covariate.

\section{Blood collection}

Blood samples were collected following an overnight fast or in the afternoon after no more than a light fat-free breakfast eaten before 08.00 hours $^{(14)}$. Cholesterol and TAG were measured in a centrifugal analyser by enzymic colorimetric methods. HDLcholesterol was determined after dextran sulfate-magnesium chloride precipitation of non-HDL-cholesterol ${ }^{(14)}$.

\section{Covariates}

Employment grade within the Civil Service was used as the measure of adult socio-economic position ${ }^{(27)}$. Weight and height were measured in standardised fashion with participants dressed in a cloth gown and underclothes. Smoking habit (never/former/current) and leisure time physical activity (hours of moderate/hours of vigorous activity per week) was self-reported. Alcohol intake (in $\mathrm{g} / \mathrm{d}$ ) was calculated from the FFQ. Blood pressure was measured twice after 5 min rest with the Hawksley random-zero sphygmomanometer at the phase 3 research clinic and the mean value taken $(\mathrm{mmHg})$.

\section{Statistical analysis}

Dietary patterns were determined using RRR techniques as previously described by Hoffmann et al. ${ }^{(5)}$ and applied in a number of recent studies of dietary patterns and chronic disease $^{(5-9,11,13)}$. RRR determines factors from food intake data that maximise the explained variation in the intermediate markers that are hypothesised to be related to the health outcome. The resulting dietary pattern scores are used in epidemiological analysis.

Total cholesterol, HDL-cholesterol and TAG were used as intermediate markers or response variables based on the established relationships with $\mathrm{CHD}^{(28-31)}$. The number of dietary patterns extracted using RRR analysis is determined

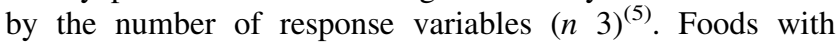
absolute factor loadings $>0.20$ were used to describe the dietary pattern but all foods contributed to calculation of the dietary pattern score ${ }^{(5,9,12,13)}$. To investigate robustness of the dietary patterns, we randomly split the cohort five times and repeated the RRR analysis in half of the cohort (i.e. the analysis was repeated five times). The mean Spearman correlation coefficient between these dietary pattern factor loadings and the original factor loadings was 0.98, 0.93 and 0.90 for patterns 1,2 and 3 , respectively. Dietary patterns were analysed using food intakes adjusted for EI, sex and employment grade using the residual method ${ }^{(32)}$. The resulting dietary patterns were similar (data not shown), and the unadjusted patterns were used in the analysis with incidence of CHD. Sex-specific quartiles of the dietary pattern scores were calculated. 
Cox proportional hazards regression was conducted using follow-up time as the time variable using Stata 9.0 (StataCorp LP, College Station, TX, USA). Males and females were combined for analysis, as models including an interaction term indicated that there was no sex interaction. Initial models were adjusted for age, sex, energy misreporting and ethnicity $^{(3)}$. Further adjustments were made for employment grade, health behaviours (smoking, alcohol and physical activity), blood pressure and BMI. $P$ values $<0.05$ were considered to be significant.

\section{Results}

Three dietary patterns were extracted explaining 4.2, 1.7 and $0.3 \%$ of the variation in response variables (Table 1). Dietary pattern 1 explained $7.14 \%$ of variation in HDL-cholesterol and $5.3 \%$ of variation in TAG while dietary pattern 2 explained $3.5 \%$ of variation in total cholesterol. As dietary pattern 3 explained little variation in responses, further results are not presented. Dietary pattern 1 was characterised by higher consumption of white bread, fried potatoes, sugar in tea and coffee, burgers and sausages, soft drinks, and lower consumption of French dressing, vinaigrette and other vegetables (Table 2). A higher score on dietary pattern 1 was associated with higher total cholesterol, lower HDL-cholesterol and higher TAG. Dietary pattern 2 was characterised by higher consumption of red meat, cabbage, Brussels sprouts and cauliflower, and lower consumption of wholemeal bread, jam, marmalade and honey, tofu and soya, buns, cakes, pastries, fruit pies and polyunsaturated margarine. A higher score on dietary pattern 2 was associated with higher total cholesterol and higher TAG.

After 83536 person-years of follow-up, 243 incident coronary events were identified (Table 3 ). For dietary pattern 1, a higher dietary pattern score was associated with increased risk of CHD (model 1, adjusted for age, sex and energy misreporting, ethnicity; $P$ for trend $<0.0001)$. This relationship was attenuated after adjustment for employment grade (model 2) and health behaviours (model 3, smoking, alcohol and physical activity), but remained significant even after further adjustment for blood pressure and BMI (model 6, hazard ratio for top quartile: $1.57 ; 95 \%$ CI 1.08, 2.27). Further adjustment for dietary patterns 2 and 3 did not affect the findings (hazard ratio for top quartile: 1.57; $95 \%$ CI 1.08, 2.27; data not shown). Dietary pattern 2 showed a significant linear trend across quartiles with a higher dietary pattern score also associated with increased risk of CHD (model 3, adjusted for age, sex and energy misreporting, ethnicity, employment grade, smoking, alcohol and physical activity; $P<0 \cdot 0001)$. However, this relationship was no longer significant after further adjustment for BMI and blood pressure. Dietary pattern 3 was not associated with CHD (hazard ratio for top quartile: $0.90 ; 95 \%$ CI $0.61,1.31$, adjusted for age, sex, energy misreporting, ethnicity and employment grade; data not shown).

\section{Discussion}

We identified a dietary pattern, characterised by high consumption of white bread, fried potatoes, sugar in tea and coffee, burgers and sausages, and soft drinks, and lower consumption of French dressing/vinaigrette and other vegetables, that was associated with higher total cholesterol, lower HDL-cholesterol and higher TAG and was predictive of increased risk of CHD. A second dietary pattern, characterised by higher consumption of red meat, cabbage, Brussels sprouts and cauliflower, and lower consumption of wholemeal bread, jam, marmalade and honey, tofu and soya, buns, cakes, pastries, fruit pies and polyunsaturated margarine, also remained associated with increased risk of CHD after adjustment for non-dietary health behaviours and socio-economic status.

We selected serum lipids to serve as intermediate biomarkers of risk in the dietary pattern-CHD analysis. There is strong evidence from observational studies ${ }^{(28)}$, cholesterollowering trials ${ }^{(29)}$ and genetic association studies ${ }^{(30,31)}$ that blood lipids are causal factors for disease. We found mean differences in total cholesterol, HDL-cholesterol and TAG of $0 \cdot 15,0.18$ and $0.37 \mathrm{mmol} / 1$ across quartiles of dietary pattern score 1 , corresponding to a two-fold higher disease rate in the base model. The impact of diet appeared little confounded by associated health behaviours. Adjustments for employment grade, a powerful indicator of adult socio-economic status, as well as smoking, alcohol and physical activity reduced the observed effect by $15 \%$.

Dietary pattern 1 was strongly associated with lower HDLcholesterol and higher TAG levels. The relationship between dietary pattern 1 and CHD risk was attenuated by inclusion of BMI in the model. This suggests that this dietary pattern is a determinant of the metabolic syndrome risk profile. Additional attenuation by the inclusion of blood pressure supports this interpretation. Further work is required to directly investigate the impact of this dietary pattern on risk of the metabolic syndrome.

Previous RRR studies of diet and CHD have used a range of intermediate markers including HDL-cholesterol, LDL-choles-

Table 1. Explained variation (\%) in food intakes and responses of each dietary pattern as assessed using reduced rank regression and correlations between the dietary pattern scores and responses

\begin{tabular}{|c|c|c|c|c|c|c|c|c|}
\hline \multirow[b]{2}{*}{ Dietary pattern } & \multicolumn{5}{|c|}{ Explained variation (\%) } & \multicolumn{3}{|c|}{$\begin{array}{l}\text { Correlations between the dietary pattern scores } \\
\text { and the response variables (blood lipids) }\end{array}$} \\
\hline & $\begin{array}{l}\text { Food intakes } \\
\text { (total) }\end{array}$ & $\begin{array}{l}\text { Responses } \\
\text { (total) }\end{array}$ & Total cholesterol & HDL-cholesterol & TAG & Total cholesterol & HDL-cholesterol & TAG \\
\hline 1 & 3.65 & $4 \cdot 19$ & 0.14 & $7 \cdot 14$ & $5 \cdot 28$ & 0.04 & -0.27 & 0.23 \\
\hline 2 & $2 \cdot 19$ & 1.67 & 3.51 & 0.88 & 0.61 & 0.19 & 0.09 & 0.08 \\
\hline 3 & $2 \cdot 07$ & 0.32 & 0.28 & 0.25 & 0.44 & -0.05 & 0.05 & 0.07 \\
\hline Total & 7.91 & $6 \cdot 18$ & 3.93 & $8 \cdot 27$ & $6 \cdot 33$ & - & - & - \\
\hline
\end{tabular}


Table 2. Intakes of the key foods and response variables across sex-specific quartiles of dietary pattern scores (Mean values with their standard errors for 7314 subjects)

\begin{tabular}{|c|c|c|c|c|c|c|c|c|c|c|}
\hline & \multirow[b]{2}{*}{ Factor loading } & \multicolumn{2}{|c|}{ Quartile 1} & \multicolumn{2}{|c|}{ Quartile 2} & \multicolumn{2}{|c|}{ Quartile 3} & \multicolumn{2}{|c|}{ Quartile 4} & \multirow[b]{2}{*}{$P$ for trend } \\
\hline & & Mean & SE & Mean & SE & Mean & SE & Mean & SE & \\
\hline \multicolumn{11}{|l|}{ Dietary pattern 1} \\
\hline \multicolumn{11}{|l|}{ Food groups (g/d) } \\
\hline \multicolumn{11}{|l|}{ Direct associations } \\
\hline White bread & 0.274 & $9 \cdot 84$ & 0.47 & $15 \cdot 08$ & 0.56 & $26 \cdot 78$ & 0.84 & $53 \cdot 25$ & $1 \cdot 22$ & $<0.0001$ \\
\hline Fried potatoes & 0.258 & $28 \cdot 19$ & 0.59 & $36 \cdot 65$ & 0.68 & $45 \cdot 17$ & 0.76 & $64 \cdot 48$ & $1 \cdot 04$ & $<0.0001$ \\
\hline Sugar in tea and coffee & 0.255 & $2 \cdot 65$ & 0.16 & $4 \cdot 41$ & 0.21 & $7 \cdot 97$ & 0.28 & $14 \cdot 76$ & 0.34 & $<0.0001$ \\
\hline Burgers and sausages & 0.222 & $4 \cdot 45$ & 0.09 & $5 \cdot 46$ & $0 \cdot 10$ & $6 \cdot 81$ & $0 \cdot 14$ & $9 \cdot 60$ & $0 \cdot 20$ & $<0.0001$ \\
\hline Soft drinks & $0 \cdot 217$ & $32 \cdot 25$ & 1.48 & $47 \cdot 62$ & 1.68 & $63 \cdot 29$ & $2 \cdot 12$ & $119 \cdot 17$ & 3.90 & $<0.0001$ \\
\hline \multicolumn{11}{|l|}{ Inverse associations } \\
\hline French dressing, vinaigrette & -0.246 & $3 \cdot 36$ & 0.10 & 1.53 & 0.05 & 0.96 & 0.04 & 0.67 & 0.03 & $<0.0001$ \\
\hline Other vegetables & -0.205 & $29 \cdot 64$ & 0.55 & $20 \cdot 39$ & 0.36 & $17 \cdot 21$ & 0.32 & $14 \cdot 57$ & 0.30 & $<0.0001$ \\
\hline \multicolumn{11}{|l|}{ Response variables } \\
\hline Total cholesterol (mmol/l) & & $6 \cdot 37$ & 0.03 & $6 \cdot 50$ & 0.03 & $6 \cdot 52$ & 0.03 & $6 \cdot 52$ & 0.03 & $<0.0001$ \\
\hline HDL-cholesterol (mmol/l) & & 1.53 & 0.01 & 1.46 & 0.01 & $1 \cdot 40$ & 0.01 & $1 \cdot 35$ & 0.01 & $<0.0001$ \\
\hline TAG $(\mathrm{mmol} / \mathrm{l})$ & & $1 \cdot 24$ & 0.02 & 1.43 & 0.02 & 1.50 & 0.02 & 1.61 & 0.02 & $<0.0001$ \\
\hline \multicolumn{11}{|l|}{ Dietary pattern 2} \\
\hline \multicolumn{11}{|l|}{ Food groups (g/d) } \\
\hline \multicolumn{11}{|l|}{ Direct associations } \\
\hline Red meat & 0.243 & $33 \cdot 35$ & 0.60 & 40.49 & 0.66 & $45 \cdot 46$ & 0.67 & $60 \cdot 92$ & 1.05 & $<0.0001$ \\
\hline Cabbage, Brussels sprouts and cauliflower & 0.237 & $30 \cdot 35$ & 0.55 & $32 \cdot 76$ & 0.59 & $36 \cdot 13$ & 0.63 & 51.58 & 0.99 & $<0.0001$ \\
\hline \multicolumn{11}{|l|}{ Inverse associations } \\
\hline Wholemeal bread & -0.318 & $82 \cdot 75$ & 1.67 & 46.96 & $1 \cdot 29$ & 32.51 & 1.00 & 23.95 & 0.85 & $<0.0001$ \\
\hline Jam, marmalade and honey & -0.286 & $18 \cdot 79$ & 0.52 & $10 \cdot 17$ & 0.30 & $7 \cdot 88$ & $0 \cdot 25$ & $5 \cdot 61$ & $0 \cdot 20$ & $<0.0001$ \\
\hline Tofu and soya & -0.250 & $6 \cdot 07$ & 0.24 & $3 \cdot 17$ & 0.06 & $2 \cdot 90$ & 0.04 & $2 \cdot 80$ & 0.04 & $<0.0001$ \\
\hline Buns, cakes, pastries, fruit pies & -0.231 & $56 \cdot 86$ & $1 \cdot 17$ & 41.92 & 0.92 & $34 \cdot 84$ & 0.73 & 28.59 & 0.64 & $<0.0001$ \\
\hline Polyunsaturated margarine & -0.213 & $14 \cdot 39$ & 0.39 & $9 \cdot 37$ & 0.29 & $7 \cdot 35$ & 0.25 & $5 \cdot 38$ & 0.22 & $<0.0001$ \\
\hline \multicolumn{11}{|l|}{ Response variables } \\
\hline Total cholesterol (mmol/l) & & $6 \cdot 18$ & 0.03 & 6.42 & 0.03 & $6 \cdot 56$ & 0.03 & $6 \cdot 75$ & 0.03 & $<0.0001$ \\
\hline HDL-cholesterol (mmol/l) & & 1.43 & 0.01 & 1.43 & 0.01 & 1.42 & 0.01 & 1.45 & 0.01 & 0.34 \\
\hline TAG (mmol/l) & & $1 \cdot 31$ & 0.02 & 1.42 & 0.02 & 1.50 & 0.02 & 1.55 & 0.02 & $<0.0001$ \\
\hline
\end{tabular}

${ }^{\star}$ Adjusted for age and ethnicity.

terol, lipoprotein(a), C-peptide, C-reactive $\operatorname{protein}^{(7)}$, plasma homocysteine, folate, vitamin $\mathrm{B}_{12}^{(8)}$ and fat, carbohydrate and fibre density ${ }^{(10)}$. Despite differences in the intermediate markers, there are some similarities in the foods identified which were associated with increased risk of CVD such as potatoes and fried potatoes ${ }^{(8,9)}$ and processed meat ${ }^{(9)}$ and in the foods that were shown to be associated with decreased risk of CVD, namely, whole grains ${ }^{(10)}$ and vegetables ${ }^{(7-10)}$. Similarly, using cluster analysis to identify dietary patterns in the Whitehall II study, Brunner et al. ${ }^{(3)}$ identified a healthy dietary cluster that was characterised by higher intakes of wholemeal bread, fruits, vegetables and polyunsaturated margarine and lower intakes of red meat which was associated with a lower risk of CHD. Previous RRR studies have not

Table 3. CHD across quartiles of dietary pattern score

(Hazard ratios and $95 \%$ confidence intervals for 7314 subjects)

\begin{tabular}{|c|c|c|c|c|c|c|c|c|c|}
\hline & & \multirow[b]{2}{*}{ Quartile $1 \mathrm{HR}$} & \multicolumn{2}{|c|}{ Quartile 2} & \multicolumn{2}{|c|}{ Quartile 3} & \multicolumn{2}{|c|}{ Quartile 4} & \multirow[b]{2}{*}{$P$ for trend } \\
\hline & & & $\mathrm{HR}$ & $95 \% \mathrm{Cl}$ & $\mathrm{HR}$ & $95 \% \mathrm{Cl}$ & $\mathrm{HR}$ & $95 \% \mathrm{Cl}$ & \\
\hline \multicolumn{10}{|c|}{ Dietary pattern 1} \\
\hline Model 1 & Age, sex, energy misreporting, ethnicity & 1.00 & 1.09 & $0.74,1.60$ & 0.96 & $0.65,1.43$ & $2 \cdot 01$ & $1.41,2.85$ & $<0.0001$ \\
\hline Model 2 & +Employment grade & 1.00 & 1.07 & $0.73,1.57$ & 0.93 & $0.62,1.39$ & 1.91 & $1.33,2.74$ & $<0.0001$ \\
\hline Model 3 & +Smoking, alcohol, physical activity & 1.00 & 1.07 & $0.73,1.57$ & 0.91 & $0.61,1.36$ & 1.81 & $1 \cdot 26,2 \cdot 62$ & $<0.0001$ \\
\hline Model 4 & +Blood pressure & 1.00 & 1.05 & $0.71,1.54$ & 0.88 & $0.59,1.32$ & $1 \cdot 72$ & $1 \cdot 19,2.49$ & $<0.0001$ \\
\hline Model 5 & +BMI (without blood pressure) & 1.00 & 1.03 & $0.70,1.52$ & 0.86 & $0.57,1.29$ & 1.61 & $1.11,2.33$ & 0.002 \\
\hline Model 6 & +Blood pressure and BMI & 1.00 & 1.02 & $0.69,1.50$ & 0.83 & $0.56,1.25$ & 1.57 & $1.08,2 \cdot 27$ & 0.003 \\
\hline \multicolumn{10}{|c|}{ Dietary pattern 2} \\
\hline Model 1 & Age, sex, energy misreporting, ethnicity & 1.00 & 0.90 & $0.60,1.36$ & 1.30 & $0.89,1.91$ & 1.44 & $0.99,2.09$ & 0.007 \\
\hline Model 2 & +Employment grade & 1.00 & 0.92 & $0.61,1.39$ & 1.32 & $0.90,1.94$ & 1.45 & $1 \cdot 00,2 \cdot 10$ & 0.006 \\
\hline Model 3 & +Smoking, alcohol, physical activity & 1.00 & 0.90 & $0.59,1.36$ & 1.25 & $0.85,1.85$ & 1.31 & $0.89,1.93$ & 0.039 \\
\hline Model 4 & +Blood pressure & 1.00 & 0.88 & $0.58,1.33$ & $1 \cdot 20$ & $0.82,1.77$ & 1.25 & $0.85,1.85$ & 0.066 \\
\hline Model 5 & +BMI (without blood pressure) & 1.00 & 0.88 & $0.58,1.33$ & $1 \cdot 18$ & $0.80,1.74$ & $1 \cdot 20$ & $0.82,1.77$ & 0.132 \\
\hline Model 6 & +Blood pressure and BMI & 1.00 & 0.86 & $0.57,1.31$ & $1 \cdot 15$ & $0.78,1.69$ & $1 \cdot 17$ & $0.79,1.72$ & 0.171 \\
\hline
\end{tabular}

HR, hazard ratio. 
identified soft drinks in their dietary patterns although recent research has identified associations between soft drink consumption and risk factors for CVD such as obesity, high blood pressure, abnormal glucose and low HDL-cholesterol, which is consistent with our findings ${ }^{(33)}$.

Strengths of the present study include the large sample size, its prospective nature and the rigorous methods of outcome ascertainment. While Hoffmann et al. ${ }^{(7)}$ also incorporated HDL- and LDL-cholesterol as dependent variables, as well as a range of intermediate markers, the study was based on a case-control design which has potential for recall bias and the biomarker measurements used as the intermediate markers were measured after the diagnosis of disease. In addition, their study only included women. Nettleton et al. ${ }^{(9)}$ conducted a cross-sectional study investigating carotid intima media thickness rather than coronary events.

Foods identified in the dietary pattern may be indicators of other foods with which they are consumed. For example, salad dressing is not consumed alone and was correlated with salad vegetables in the present study (data not shown); its presence in the dietary pattern is likely to reflect consumption with salad vegetables. When investigated separately, salad dressing was not associated with CHD risk; however, as the effects of a total dietary pattern may be additive, a causal role for salad dressing cannot be ruled out. Similarly, a dietary pattern containing cruciferous vegetables (such as Brussels sprouts and cauliflower) was associated with an increased risk of CHD in this analysis. Importantly, when the individual foods were investigated separately, none of the foods showed significant associations with risk of CHD (data not shown).

Research into dietary patterns aims to characterise the whole diet in combination, providing a summary measure of dietary exposure and capture complex behaviours and potentially interactive and antagonistic effects among nutrients that might impact upon health outcomes. RRR analysis is a new statistical method which has been applied to dietary pattern research. It combines data-driven methods with use of a priori knowledge of diet-disease relationships. RRR determines dietary patterns that explain the greatest variation in the intermediate markers and responses rather than explaining variation in food intakes and, therefore, is particularly suited to identifying and confirming pathways through which dietary factors influence disease development in studies where intermediate biomarkers of exposure or disease are available ${ }^{(12)}$.

Additional work with the RRR techniques is required to determine the importance of dietary patterns extracted subsequent to the first pattern, which by definition explains the greatest variation in biomarkers. In the present study, the third dietary pattern explained very little additional variation in any of the response variables, and was not predictive of CHD. However, previous work by Hoffmann et al. ${ }^{(5)}$ showed that only the fourth dietary pattern extracted was associated with the risk of type 2 diabetes, despite explaining a small proportion of the variation in comparison with the first three dietary patterns that were extracted. Further work is required is determine the importance of extracting and investigating these subsequent patterns. A potential weakness of the RRR approach is the cross-sectional nature of underlying RRR dietary pattern analysis; however, in our analysis we excluded participants who had a previous history of $\mathrm{CHD}$ in order to reduce the impact of changes in dietary behaviour due to pre-existing disease. In addition, further work is also required to determine whether the optimal use of the RRR method involves using biomarkers that reflect one disease progression pathway, with individual pathways modelled separately or whether there is an advantage to using multiple biomarker pathways in the same regression modelling ${ }^{(11,12)}$.

The dietary patterns explained $3.9 \%$ of the variation in total cholesterol, $7.1 \%$ of the variation in HDL-cholesterol and $5.3 \%$ of the variation in TAG. These results are comparable with other studies using RRR methods where biomedical risk factors have been used as the response variable ${ }^{(7,11)}$. Studies using intakes of nutrient have tended to explain higher variation in those responses ${ }^{(5,6,13)}$. However, this is unsurprising as bloods lipids are a more remote response variable than nutrient intakes.

A range of socio-demographic factors (age, sex, ethnicity, employment grade), health behaviours (smoking, physical activity, alcohol) and other risk factors (blood pressure and BMI) were investigated as confounders and shown to attenuate the relationship between the dietary pattern and CHD, although the relationship remained significant. However, residual confounding cannot be ruled out due to the potential for measurement error among the covariates. BMI and blood pressure were included in the final regression models and shown to attenuate the relationship between dietary patterns and risk of CHD. This finding is consistent with the mediating role of these risk factors. The fully adjusted models may therefore underestimate the effect of $\operatorname{diet}^{(34)}$, while model 3, adjusted for energy misreporting, socio-demographic factors and non-dietary health behaviours, may reflect the role of diet in CHD causation operating through multiple pathways.

This research highlights that certain dietary patterns are strongly associated with blood lipids and risk of CHD, and adds to the evidence that influences on HDL-cholesterol and TAG levels are important in the dietary prevention of CHD. While the dietary pattern we identified may in part act though obesity and increased blood pressure, it appeared to have independent effects on the risk of CHD. Our findings are consistent with the operation of multiple dietary pathways influencing CHD risk. The RRR method is an advance in methodology, able to identify and confirm mechanisms through which diet may influence disease risk.

\section{Acknowledgements}

The Whitehall II study has been supported by grants from the UK Medical Research Council, British Heart Foundation, Health and Safety Executive, Department of Health, National Heart Lung and Blood Institute (HL36310), National Institute on Aging (AG13196), Agency for Health Care Policy Research (HS06516) and the MacArthur Foundation Research Network on Socio-economic Status and Health. No funding source had direct influence over the design, conduct or reporting of the present study. S. A. M. is supported by a National Health \& Medical Research Council (NHMRC) Public Health Postdoctoral Fellowship and an NHMRC Travelling Award for Research Training.

S. A. M. contributed to the development of the study hypothesis, reviewed the literature, conducted the statistical analysis, provided interpretation of results, and drafted 
and edited the manuscript. G. D. M. contributed to the development of the study hypothesis, guided the statistical analysis, provided advice regarding interpretation of the results, and assisted with drafting and editing of the manuscript. E. J. B. was responsible for the study design, data collection, provided advice regarding interpretation of the results and edited the manuscript.

None of the contributing authors had a conflict of interest.

\section{References}

1. Ness AR \& Powles JW (1997) Fruit and vegetables, and cardiovascular disease: a review. Int J Epidemiol 26, 1-13.

2. Newby PK \& Tucker KL (2004) Empirically derived eating patterns using factor or cluster analysis: a review. Nutr Rev 62, 177-203.

3. Brunner EJ, Mosdøl A, Witte DR, et al. (2008) Dietary patterns and 15 -y risks of major coronary events, diabetes, and mortality. Am J Clin Nutr 87, 1414-1421.

4. Schulze MB \& Hoffmann K (2006) Methodological approaches to study dietary patterns in relation to risk of coronary heart disease and stroke. Br J Nutr 95, 860-869.

5. Hoffmann K, Schulze MB, Schienkiewitz A, et al. (2004) Application of a new statistical method to derive dietary patterns in nutritional epidemiology. Am J Epidemiol 159, 935-944.

6. Schulz M, Nothlings U, Hoffmann K, et al. (2005) Identification of a food pattern characterized by high-fiber and low-fat food choices associated with low prospective weight change in the EPIC-Potsdam cohort. $J$ Nutr 135, 1183-1189.

7. Hoffmann K, Zyriac BC, Boeing H, et al. (2004) A dietary pattern derived to explain biomarker variation is strongly associated with risk of coronary artery disease. Am J Clin Nutr 80, 633-640.

8. Weikert C, Hoffmann K, Dierkes J, et al. (2005) A homocysteine metabolism-related dietary pattern and the risk of coronary heart disease in two independent German study populations. J Nutr 135, 1981-1988.

9. Nettleton JA, Steffen LM, Schulze MB, et al. (2007) Associations between markers of subclinical atherosclerosis and dietary patterns derived by principal components analysis and reduced rank regression in the Multi-Ethnic Study of Atherosclerosis (MESA). Am J Clin Nutr 85, 1615-1625.

10. Drogan D, Hoffmann K, Schulz M, et al. (2007) A food pattern predicting prospective weight change is associated with risk of fatal but not with nonfatal cardiovascular disease. J Nutr 137, 1961-1967.

11. Schulze MB, Hoffmann K, Manson JE, et al. (2005) Dietary pattern, inflammation, and incidence of type 2 diabetes in women. Am J Clin Nutr 82, 675-684.

12. Heidemann C, Hoffman K, Spranger J, et al. (2005) Dietary pattern protective against type 2 diabetes in the European Prospective Investigation into Cancer and Nutrition (EPIC)-Potsdam Study Cohort. Diabetologia 48, 1126-1134.

13. Hoffmann K, Boeing H, Boffetta P, et al. (2005) Comparison of two statistical approaches to predict all-cause mortality by dietary patterns in German elderly subjects. Br J Nutr 93, 709-716.

14. Brunner EJ, Marmot MG, Nanchahal K, et al. (1997) Social inequality in coronary risk: central obesity and the metabolic syndrome. Evidence from the Whitehall II study. Diabetologia 40, $1341-1349$.

15. Marmot MG \& Brunner EJ (2005) Cohort profile: the Whitehall II study. Int J Epidemiol 34, 251-256.

16. Brunner EJ, Wunsch H \& Marmot MG (2001) What is an optimal diet? Relationship of macronutrient intake to obesity, glucose tolerance, lipoprotein cholesterol levels and the metabolic syndrome in the Whitehall II study. Int $J$ Obes Relat Metab Disord 25, 45-53.
17. Black AE (2000) Critical evaluation of energy intake using the Goldberg cut-off for energy intake: basal metabolic rate. A practical guide to its calculation, use and limitations. Int J Obes 24, 1119-1130.

18. Schofield WN, Schofield C \& James WPT (1985) Basal metabolic rate. Hum Nutr Clin Nutr 39C, Suppl. 1, 1-96.

19. Ainsworth BE, Haskell WL, Whitt MC, et al. (2000) Compendium of physical activities: an update of activity codes and MET intensities. Med Sci Sports Exerc 32, Suppl. 9, S498-S504.

20. Nettleton JA, Steffen LM, Mayer-Davis EJ, et al. (2006) Dietary patterns are associated with biochemical markers of inflammation and endothelial activation in the Multi-Ethnic Study of Atherosclerosis (MESA). Am J Clin Nutr 83, 1369-1379.

21. Van Dam RM, Grievink L, Ocke MC, et al. (2003) Patterns of food consumption and risk factors for cardiovascular disease in the general Dutch population. Am J Clin Nutr 77, 1156-1163.

22. Henderson L, Gregory J \& Swan G (2002) National Diet and Nutrition Survey: adults aged 19 to 64 years. Volume 1. Types and Quantities of Foods Consumed. Norwich: HM Stationery Office.

23. Williams DE, Prevost AT, Whichelow MJ, et al. (2000) A crosssectional study of dietary patterns with glucose intolerance and other features of the metabolic syndrome. $\mathrm{Br} J$ Nutr $\mathbf{8 3}$, 257-266.

24. Van Dam RM, Rimm EB, Willett WC, et al. (2002) Dietary patterns and risk for type 2 diabetes mellitus in U.S. men. Ann Inter Med 136, 201-209.

25. Esmaillzadeh A, Kimiagar M, Mehrabi Y, et al. (2007) Dietary patterns, insulin resistance and prevalence of the metabolic syndrome. Am J Clin Nutr 85, 910-998.

26. Emberson JR, Shaper AG, Wannamethee SG, et al. (2005) Alcohol intake in middle age and risk of cardiovascular disease and mortality: accounting for intake variation over time. Am J Epidemiol 161, 856-863.

27. Martikainen P, Brunner E \& Marmot M (2003) Socioeconomic differences in dietary patterns among middle-aged men and women. Soc Sci Med 56, 1397-1410.

28. Lewington S, Whitlock G, Clarke R, et al. (2007) Blood cholesterol and vascular mortality by age, sex, and blood pressure: a meta-analysis of individual data from 61 prospective studies with 55000 vascular deaths. Lancet 370, 1829-1839.

29. Baigent C, Keech A, Kearney PM, et al. (2005) Efficacy and safety of cholesterol-lowering treatment: prospective meta-analysis of data from 90056 participants in 14 randomised trials of statins. Lancet 366, 1267-1278.

30. Wittrup HH, Tybjaerg-Hansen A \& Nordestgaard BG (1999) Lipoprotein lipase mutations, plasma lipids and lipoproteins, and risk of ischemic heart disease. A meta-analysis. Circulation 99, 2901-2907.

31. Wittrup HH, Andersen RV, Tybjaerg-Hansen A, et al. (2006) Combined analysis of six lipoprotein lipase genetic variants on triglycerides, high-density lipoprotein, and ischemic heart disease: cross-sectional, prospective, and case-control studies from the Copenhagen City Heart Study. J Clin Endocrinol Metab 91, 1438-1445.

32. Willett W \& Stampfer M (1998) Implications of total energy intake for epidemiologic analyses. In Nutritional Epidemiology, pp. 101-147 [W Willett, editor]. New York: Oxford University Press.

33. Dhingra R, Sullivan L, Jacques PF, et al. (2007) Soft drink consumption and risk of developing cardiometabolic risk factors and the metabolic syndrome in middle-aged adults in the community. Circulation 116, 480-488.

34. Srinath Reddy K \& Katan MB (2004) Diet, nutrition and the prevention of hypertension and cardiovascular diseases. Public Health Nutr 7, 167-186. 